\title{
Téoros
}

Revue de recherche en tourisme

\section{Tourisme et congrès : deux phénomènes distincts}

\section{Louis Jolin}

Volume 4, numéro 3, novembre 1985

L'essor de l'industrie des congrès

URI : https://id.erudit.org/iderudit/1080783ar

DOI : https://doi.org/10.7202/1080783ar

Aller au sommaire du numéro

Éditeur(s)

Université du Québec à Montréal

ISSN

0712-8657 (imprimé)

1923-2705 (numérique)

Découvrir la revue

Citer cet article

Jolin, L. (1985). Tourisme et congrès : deux phénomènes distincts. Téoros, 4(3),

2-2. https://doi.org/10.7202/1080783ar d'utilisation que vous pouvez consulter en ligne.

https://apropos.erudit.org/fr/usagers/politique-dutilisation/ 


\title{
Tourisme et congrès: deux phénomènes distincts
}

\author{
par Louis Jolin *
}

Les congressistes sont-ils des touristes? Les congrès constituent-ils une composante de I'industrie du tourisme? Pouvons-nous parler d'un tourisme de congres a l'instar d'un tourisme d'agrément? Ou tourisme et congrès ne sont-ils pas plutôt deux réalités distinctes qui entretiennent cependant entre elles des rapports très étroits?

Ces questions posent le problème de la définition du tourisme. Et il s'agit d'un problème sérieux dont la résolution peut avoir des conséquences non négligeables sur les stratégies de développement et de promotion.

\section{Les paradigmes}

Dans une trẻs intéressante communication présentée en 1984 lors du 4 e Congrès canadien de recherche en loisir, le professeur Jean Stafford souligne limportance des paradigmes dans la recherche scientifique. Se référant à Thomas Kuhn, il rappelle qu'un paradigme est un cadre de pensée. d'idées maitresses et de façons de travailler qui déterminent la perception des problëmes et proposent des manières de les résoudre. " Et, si la "science du tourisme" est en fait préparadigmatique, on $y$ retrouve du moins l'émergence de grands paradigmes quil représentent une certaine vision du tourisme, chaque paradigme s'articulant autour de définitions du tourisme plus ou moins strictes et limitées de I'objet d'étude.

Sans entrer dans une présentation détaillée de la communication de Jean Stafford, soulignons, à titre d'exemple, que le paradigme économico-spatial du tourisme s'intéresse plus particulièrement aux aspects financiers du tourisme et aux déplacements physiques des voyages. Dans la dimension spatiale de ce paradigme, "on perçoit le touriste surtout comme un utilisateur d'un moven quelconque de transport". ${ }^{21}$ Pas étonnant que dans cette approche, l'on soit tenté d'intégrer dans la définition du tourisme tout le phénomène des congrès. Les congrès ne font-ils pas l'objet d'un comportement

"Louis Jolin est direxteur du module de gestion et intervention touristiques de I"UOAM ot directeur de la revue Téros, d'achat et ne sont-ils pas très souvent l'occasion d'un déplacement d'un point géographique a un autre? Un autre paradigme dit "culturaliste" percoit le tourisme comme l'expression d'attentes, d'attitudes et de motivations; il s'intéresse aux valeurs dans la mesure où celles-ci influencent les rapports entre l'individu, les structures institutionnelles et l'ensemble des processus psycho-sociaux. La définition du tourisme, selon cette approche, est plus complexe, ne se rósume pas aux seuls déplacements physiques ou aux aspects financiers du phénomène et se distingue plus nettement des définitions de réalités avoisinantes telles que les congres...

Quel paradigme devrait-on choisir? Jean Stafford mentionne à juste titre qu "isils sont en compétition et que les gens qui ont choisi consciemment ou inconsciemment un paradigme doivent en général défendre ce paradigme face aux membres de la communauté scientifique."

\section{Ma position}

Je défends alors ma position. Je crois sincerrement qu'on noie le poisson en voulant restreindre le tourisme aux seules dimensions économiques et financiêres des déplacements de visiteurs. Une stratégie de développement et de promotion qui ne prend en compte que cet aspect ne sera mue que par le désir d'accroitre le nombre de visiteurs, le nombre de devises étrangères qu'amènent aveo eux ces visiteurs indépendamment des conséquences de ces déplacements sur les communautés d'accueil et de ce que ces visiteurs vivent comme expérience. Dans cette strategie, il est certes important de développer les congrès présentés comme une composante, parmi d'autres, du phénomène touristique. Plus il $y$ aura de congrès au Québec, plus il y aura de visiteurs qui dépenseront au Québec, ce qui ne peut être que bénéfique pour les fournisseurs de services.

Mais, pour moi, le tourisme est ả la fois plus complexe et plus spécifique que celà. Je me référe plutôt à la définition de $M$. Przeclawski qui décrit le tourisme comme "I'ensemble des phénomènes de déplace. ment temporaire et volontaire lié au changement du milieu et du rythme de la vie, et lié à la prise du contact personnel avec le milieu visité, naturel, culturel, social." ${ }^{.|4|}$ Le tourisme doit obligatoirement impliquer cette prise de contact, cette "confrontation" des cultures, celle du visiteur et celle du visité. Dans cette perspective, il faut donc distinguer congres et tourisme. Les conditions dans lesquelles se déroule un congrès dans un pays donné ne favorisent pas nécessairement pour les congressistes étrangers une prise de contact avec la réalité de ce pays et sa population. Pour que cela se produise, il faut généralement développer des activités pré et post-congrès, activités quil vont permettre aux congressistes de sortir de leur palais, de leur hôtel pour aller à la rencontre du pays et de sa population, pour faire du tourisme quoil

Celà dit, les congrès restent importants car ils sont l'occasion de la venue de nombreux visiteurs, mais encore faut-il faire de ces visiteurs des touristes. Les congrès peuvent ête pris en considération dans une stratégie de développement touristique mais ne devraient pas être confondus avec le tourisme. Les phénomenes sont voisins, souvent nécessaires l'un â l'autre tout en étant fondamentalement distincts. Alors plutôt que de parler de tourisme de congrès, je préfére nettement explorer les rapports stroits entre les congres et le tourisme. C'est un choix et je le sais en compétition avec d'autres. Qu'en pensez-vous?

\section{Notes et références}

(1) STAFFORD, Jean, Les paradigmes de la recherche en teorologie: btude, analyse et critique. texte de la communication présentée au 4 e Con grès canadien de recherche en loisir, mai 1984, p. 3. Jean Stafford cite Thomas KUHN, La structure des révolutions scientifiques, Paris, Flammation, 1972.

121 STAFFORD, Jean, op, cit. p. 8.

(3) Didem, p. 3.

(4) PRZECLAWSKI, Krysztof, Tourisme - Rencontre des cultures, Introduction à la sociologie du tourisme, Varsovie, Institut de la recherche sur la jeunesse, 1975 . p. 12. 